\title{
Vascular responsiveness in adrenalectomized rats with corticosterone replacement
}

\author{
DANIEL N. DARLINGTON, KAPIL KASHIP, LANNY C. KEIL, AND MARY F. DALLMAN \\ Department of Physiology, University of California, San Francisco, 94143; and National Aeronautics \\ and Space Administration-Ames Research Center, Moffett Field, California 94035
}

\begin{abstract}
Darlington, Daniel N., Kapil Kaship, Lanny C. Keil, AND MARy F. DALlman. Vascular responsiveness in adrenalectomized rats with corticosterone replacement. Am. J. Physiol. 256 (Heart Circ. Physiol. 25): H1274-H1281, 1989.-To determine under resting, unstressed conditions the circulating glucocorticoid concentrations that best maintain sensitivity of the vascular smooth muscle and baroreceptor responses to vasoactive agents, rats with vascular cannulas were sham-adrenalectomized (sham) or adrenalectomized (ADRX) and provided with four levels of corticosterone replacement $(\sim 100 \mathrm{mg}$ fused pellets of corticosterone: cholesterol $0,20,40$, and $80 \%$ implanted subcutaneously at the time of adrenal surgery). Changes in vascular and baroreflex responses were determined after intravenous injection of varying doses of phenylephrine and nitroglycerin with measurement of arterial blood pressure and heart rate in the conscious, chronically cannulated rats. Vascular sensitivity was decreased, and resting arterial blood pressure tended to be decreased in the adrenalectomized rats; both were restored to normal with levels of corticosterone (40\%), which also maintained body weight gain, thymus weight, and plasma corticosteroid binding globulin concentrations at normal values. The baroreflex curve generated from the sham group was different from the curves generated from the ADRX $+0,20$, and $40 \%$ groups, but not different from that of the ADRX $+80 \%$ group, suggesting that the baroreflex is maintained by higher levels of corticosterone than are necessary for the maintenance of the other variables. These data demonstrate that physiological levels of corticosterone $(40 \%$ pellet) restore vascular responsiveness, body weight, thymus weight, and transcortin levels to normal in ADRX rats, whereas higher levels $(80 \%$ pellet) are necessary for restoration of the baroreflex.
\end{abstract}

adrenalectomy; baroreflex; pressoreceptor; arterial pressure; renin; vasopressin; oxytocin; corticosteroid-binding globulin

PATIENTS WITH ADRENAL INSUFFICIENCY have been described as being hypotensive and resistant to pressor agents unless treated with adrenal steroids $(3,11,21)$. These patients are considered extremely fragile, because stimuli that can be tolerated by normal individuals may lead to cardiovascular collapse. Early studies in anesthetized rats exposed to acute surgery demonstrated that the vascular smooth muscle became very insensitive to pressor agents unless pretreated with glucocorticoids $(8$, $14,23)$. This was confirmed in anesthetized dogs and cats $(7,18)$, and the hypothesis was advanced that the cardiovascular collapse observed in individuals suffering from adrenal crises was, at least partially, due to vascular insensitivity to constrictor agents. Later studies in conscious dogs suggested that adrenalectomy impaired cardiac function $(20,24,28)$ either by the permissive action of glucocorticoids on cardiac tissue (26) or possibly by affecting the central neural components of the baroreflex. Glucocorticoid receptors have recently been found in neurons of the A1 and A2 cell groups in the brain stem medulla (10).

Adrenal steroid replacement in adrenalectomized animals has been shown to improve the health and stability of the preparation. Vascular responsiveness to norepinephrine improved after administration of corticosterone or adrenal cortical extract $(2,8,18,23)$; however, in these early studies, the doses of glucocorticoid were either high or indeterminate. It is not clear what concentrations of corticosterone improve vascular responsiveness after adrenalectomy, nor is it clear how large the cardiovascular deficit is in the unstressed, relatively healthy adrenalectomized rat.

In this study, we have examined the changes in vascular responsiveness and the baroreflex after adrenalectomy in conscious, unrestrained and unstressed rats treated with a variety of replacement doses of corticosterone. The doses were chosen to maintain plasma corticosterone concentrations in the lower quarter of the physiological range that is observed in intact rats. We compared the corticosterone levels, which corrected the changes in vascular responsiveness after adrenalectomy, with those that corrected deviations in other variables affected by adrenalectomy [plasma renin, corticosteroidbinding globulin (CBG), body weight, and thymus weight].

\section{MATERIALS AND METHODS}

General procedures. Male Sprague-Dawley rats weighing $275-350 \mathrm{~g}$ were chronically cannulated as described previously (6). Briefly, the rats were anesthetized with pentobarbital sodium $(45 \mathrm{mg} / \mathrm{kg} \mathrm{ip})$, and femoral vein (PE-50) and artery (Dural Plastics) cannulas were placed using sterile procedures. The cannulas were tunneled under the skin of the back and through a spring that was attached to the back of the neck and the top of the cage. All incisions were filled with xylocaine jelly and Polysporin (Burroughs-Wellcome) to desensitize the surgical area and to prevent infection. The rats recovered and were caged singly in a room (controlled temperature and humidity) with a 12-h on/off light cycle. All rats had 
access to food and water ad libitum.

Daily measurements of mean arterial blood pressure, heart rate, and fluid intake were made after flushing the cannulas. The arterial cannula was connected to a Statham pressure transducer and Grass amplifier and tachygraph. This was done without handling or disturbing the animals in the cage as the cannulas were manipulated from the outside.

Three days later, rats were either bilaterally adrenale tomized or sham adrenalectomized under either anesthesia by the dorsal approach. Corticosterone replacement was effected by placing fused pellets of 20,40 , or $80 \%$ corticosterone-cholesterol $(\sim 100 \mathrm{mg})$ or wax pellets under the skin of the back as described by Akana et al. (1). The rats recovered and were all given $0.5 \%$ saline to drink and food ad libitum. Saline was given to replace the sodium lost in the urine after removal of the mineralcorticoids (adrenalectomy).

All experiments were performed at least 5 days after adrenalectomy. Blood samples (whole body) were taken in the morning ( $2 \mathrm{~h}$ after lights on) and just before lights off for measurement of plasma corticosterone $(0.1 \mathrm{ml})$. Vasopressin $(1 \mathrm{ml})$, oxytocin $(1 \mathrm{ml})$, CBG $(0.1 \mathrm{ml})$, osmolality, $\mathrm{Na}^{+}, \mathrm{K}^{+}(0.25 \mathrm{ml})$, and blood volume $(0.25 \mathrm{ml})$ were determined from a morning sample. All samples were centrifuged, the plasma was separated and put on ice, and the red cells were resuspended in saline and returned to the rat (usually within $2 \mathrm{~min}$ ).

On the sixth day after adrenalectomy, vascular sensitivity was tested to varying doses of the $\alpha_{1}$-agonist phenylephrine and nitroglycerin. In the morning, arterial pressure and heart rate were monitored continuously while injections (not more than $0.1 \mathrm{ml}$ ) of phenylephrine or nitroglycerin were made through the venous cannula. The peak changes in pressure and heart rate were recorded and compared with resting pressure and heart rate. Resting measurements were taken before injection and were only considered when the rat was resting quietly on all fours. Measurements were not taken during grooming, eating, or drinking, because these maneuvers have profound effects on heart rate and blood pressure.

Hormone assays. Plasma corticosterone was measured in heat-denatured samples (29) with inter- and intraassay coefficients of variation of 8 and $10 \%$, respectively. The minimal detectable level for a $10-\mu \mathrm{l}$ sample was 0.1 $\mu \mathrm{g} / \mathrm{dl}$. Vasopressin was measured by radioimmunoassay (RIA) from bentonite-extracted plasma (27). The interand intra-assay coefficients of variation were 9 and $12 \%$, respectively. The lowest detectable level was $0.3 \mathrm{pg} / \mathrm{ml}$. Oxytocin was measured by RIA from acetone-extracted plasma (15). The inter- and intra-assay coefficients of variation were 3.2 and $6.8 \%$, respectively. The lowest detectable level was $0.8 \mathrm{pg} / \mathrm{ml}$. Plasma renin concentration (PRC) was determined by adding $100 \mu$ l of sample plasma to $900 \mu \mathrm{l}$ of nephrectomized rat plasma at $\mathrm{pH}$ 6.5. PRC was determined by generation of angiotensin I in vitro by RIA (25). The inter- and intra-assay coefficients of variation were 5.2 and $11.3 \%$, respectively. The lowest detectable level was $1 \mathrm{ng}$ angiotensin $\mathrm{I} / \mathrm{ml} \cdot 2 \mathrm{~h}$. Plasma CBG was measured by RIA (19).

Blood volume and plasma electrolytes. Blood volume was estimated by the dye dilution technique. One milligram of Evans blue dye was injected intravenously as a priming dose. Then $1 \mathrm{mg}$ of Evans blue was injected again. Plasma samples were taken before and $5 \mathrm{~min}$ after the second injection. The concentration of dye in the plasma was measured with a spectrophotometer (Gilford). Plasma osmolality and protein were measured with an osmometer (Advanced Instruments) and a hand protometer (National). Plasma $\mathrm{Na}^{+}$and $\mathrm{K}^{+}$were measured on a flame photometer (Instrumental Laboratory).

Regression analysis and statistics. Daily measurements of arterial blood pressure, heart rate, and fluid intake were analyzed by two-way analysis of variance (ANOVA) corrected for repeated measures over time. The changes in mean arterial blood pressure to injection of phenylephrine and nitroglycerin were analyzed by two-way ANOVA. The plasma levels of corticosterone were analyzed by ANOVA and after logarithmic transform, PRC, vasopressin, oxytocin, CBG, plasma protein, plasma volume, osmolality, thymus weight, and body weight in rats with different corticosterone replacement were analyzed by ANOVA. Circadian corticosterone levels, $\mathrm{K}^{+}$, and $\mathrm{Na}^{+}$ were analyzed by paired and unpaired Student's $t$ tests.

To compare the responses of heart rate with varying levels of arterial blood pressure, a stepwise polynomial regression was performed while testing the coefficient of highest power for statistical significance by $F$ test (31). The regression coefficients that were calculated for each treatment group were compared with sham and adrenalectomy treatment (ADRX) groups by $t$ test. Significance is indicated by $P<0.05$.

\section{RESULTS}

The responsiveness of the vasculature was tested by examining the changes in mean arterial blood pressure (MABP) after intravenous injections of phenylephrine and nitroglycerin (Fig. 1). Injection of phenylephrine led to significantly smaller dose-dependent increases in MABP in the ADRX group compared with the sham group, suggesting that the vasculature in the ADRX group does not have the same capacity to respond to the $\alpha_{1}$-agonist as the sham group. Also, the change in MABP after phenylephrine in the ADRX group was not as great as that in the sham group. Resting MABP in the ADRX group tended to be lower (although not significantly lower by Newman-Keuls analysis) than in the sham group. Intravenous injection of nitroglycerin led to dosedependent decreases in MABP, and the responses of the two groups were different. However, this difference was due to the lower resting MABP in the ADRX group; the changes in MABP were the same in both groups.

Changes in the responsiveness of the vascular smooth muscle to constricting and dilating agents are reflected by changes in arterial pressure. However, arterial pressure is also influenced by changes in heart rate, especially changes induced by the baroreflex. In these studies, it was found that, at any given arterial pressure above or below resting, the heart rate was not significantly different between groups (two-way ANOVA over the doses of phenylephrine and nitroglycerin). Therefore, the smaller increase in MABP in response to phenylephrine meas. 

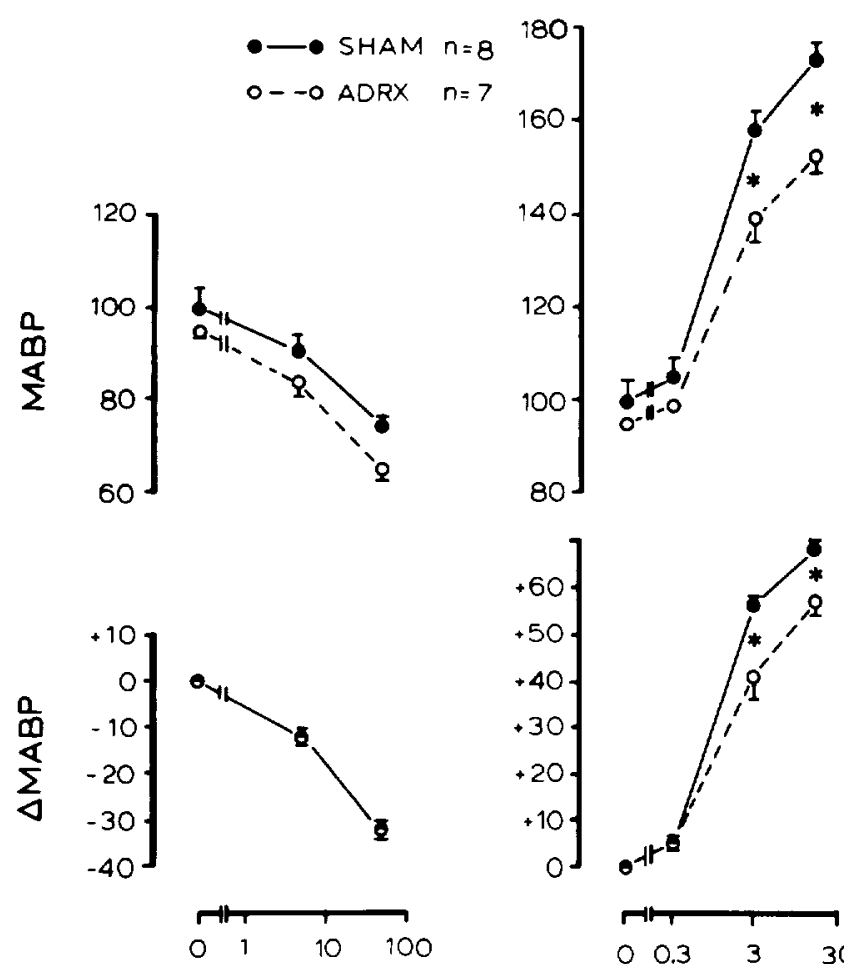

Nitroglycerin $(\mu g / r a t)$

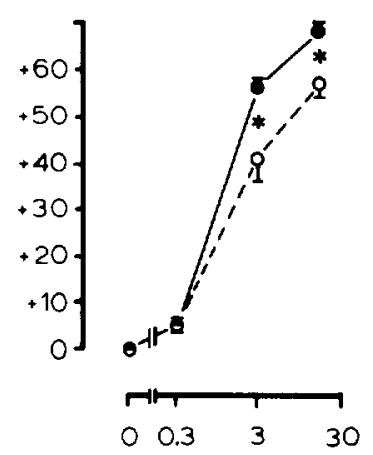

Phenylephrine ( $\mu \mathrm{g} / \mathrm{rat})$

FIG. 1. Response of mean arterial blood pressure (MABP, $\mathrm{mmHg}$ ) and change in MABP ( $\triangle \mathrm{MABP}$ ) to intravenous injections of phenylephrine or nitroglycerin in conscious nonstressed rats that were adrenalectomized (ADRX) or sham ADRX 6 days before experiment. Vehicle was injected at 0 . Concentrations are expressed in $\mu \mathrm{g} / \mathrm{rat}$, because body weights of sham and ADRX groups were not different at the time of experiment. Values are means $\pm \mathrm{SE} .{ }^{*} P<0.05$ by NewmanKeuls after two-way analysis of variance.

ured in the ADRX group was not due to a greater bradycardia (the bradycardia in the sham group was actually smaller; sham $286 \pm 12$, ADRX $312 \pm 11$ beats/ $\mathrm{min}$ ), but was more likely due to a decrease in vascular responsiveness.

Plasma corticosterone levels were measured in the morning and in the evening to verify completeness of adrenalectomy. The sham group had the expected diurnal elevation in plasma corticosterone (morning $0.5 \pm 0.1$ and evening $9.0 \pm 2.3 \mu \mathrm{g} / \mathrm{dl}$ ), whereas there was no rhythm in the adrenalectomized group (morning $0.5 \pm$ 0.2 and evening $0.5 \pm 0.1 \mu \mathrm{g} / \mathrm{dl}$ ). Morning levels of corticosterone in sham rats are not usually different from ADRX rats, because under resting conditions the pituitary-adrenal system is inactive at this time of day (5).

The vascular responsiveness to phenylephrine was impaired, and basal MABP was lower after adrenalectomy (Fig. 1, experiment 1). To determine whether glucocorticoid replacement restores basal MABP and vascular responsiveness to normal, a second experiment was performed in which pellets containing $0,20,40$, and $80 \%$ corticosterone were implanted into ADRX rats; sham controls were also included.

The response of MABP to injections of phenylephrine was significantly impaired after adrenalectomy with 0 and $20 \%$ corticosterone replacement (Fig. 2,

ADRX $+20 \%$ not shown). However, the curve generated in the $40 \%$ pellet group was not different from that in the sham group. The curve generated in the $80 \%$ pellet group was significantly different from the sham group, suggesting overreplacement by corticosterone.

The response of MABP to injections of nitroglycerin was significantly different after adrenalectomy with 0 , 20 , and $40 \%$ replacement (Fig. 2). The curve generated in the $80 \%$ pellet group was not significantly different from sham; however, the major effect of corticosterone replacement was to return basal pressure to normal. In general, lower concentrations of corticosterone corrected the change in basal MABP at the lower doses of phenylephrine and nitroglycerin, and higher concentrations of corticosterone corrected for both the lower MABP and the deficit in vascular responsiveness at the higher doses of phenylephrine and nitroglycerin.

Corticosterone pellets have been previously shown to produce stable concentrations of corticosterone in adrenalectomized rats (1). Figure 2 and Table 1 show the concentrations of corticosterone measured in the morning before the vascular sensitivity experiment in each group. All of these values are well below the circadian maximum.

The effect of ADRX and corticosterone replacement on the baroreflex was examined in the sham and ADRX + replacement groups. This reflex was measured as the heart rate recorded after changing arterial blood pressure with phenylephrine or nitroglycerin (Fig. 3). A stepwise polynomial regression was performed on each data set, testing the highest power coefficient for significance of the regression. It was found that the most significant regression for the $A D R X+0$ group was a straight line, and the most significant regression for the sham group was a polynomial with a quadratic term (Fig. 3), suggesting that adrenalectomy affected the baroreflex. Replacement of corticosterone in low doses (ADRX+20 and $+40 \%$ groups) also generated straight lines with regression coefficients ( $\alpha$ and $\beta_{1}$ ) not different from the coefficients calculated from the ADRX $+0 \%$ group (Table 2). However, the highest dose of corticosterone replacement $(\mathrm{ADRX}+80 \%)$ generated a polynomial with a quadratic term with regression coefficients $\left(\alpha, \beta_{1}\right.$, and $\left.\beta_{2}\right)$ not different from the sham group (Table 2 ), suggesting that the $80 \%$ pellet better approximated corticosterone replacement for restoration of the baroreflex to normal. On further inspection of Fig. 3, it is evident that shape of the curve was determined by the points in the upper blood pressure range. Because the larger doses of phenylephrine increased arterial pressure more in the sham and $\mathrm{ADRX}+80 \%$ groups than in the other three groups (Fig. 2), there were more points on the sham and ADRX $+80 \%$ graph above $180 \mathrm{mmHg}$. Although this part of the graph was important in determining the shape of the curve in these two groups, elimination of the points $>180 \mathrm{mmHg}$, and again performing the stepwise regression, did not change the results stated above.

The baroreflex was further analyzed by plotting the increases and decreases in arterial pressure, separately, against the changes in heart rate and calculating the slope of the linear regression. The slope can be consid- 


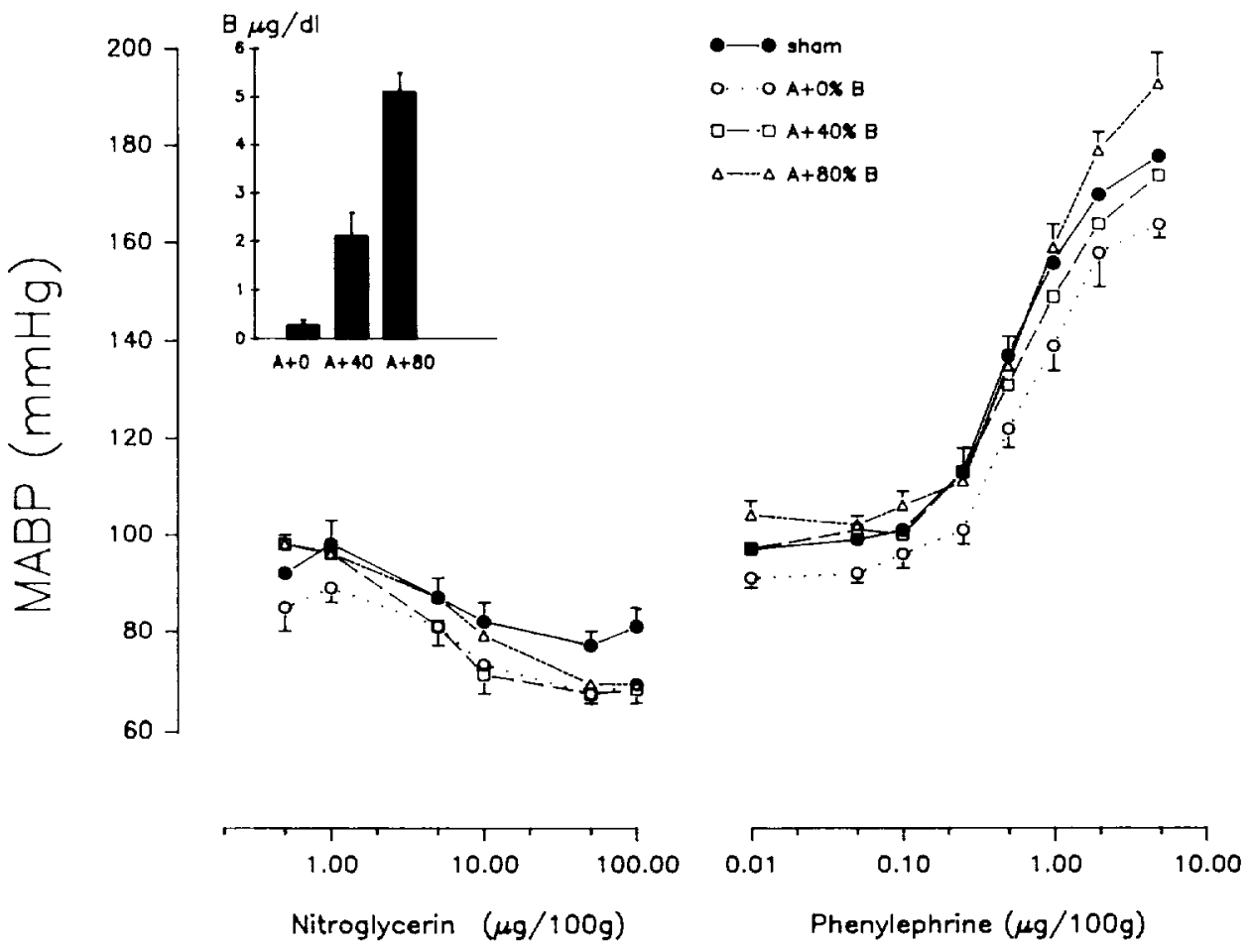

FIG. 2. Mean arterial blood pressure (MABP) after intravenous injection of nitroglycerin or phenylephrine (expressed in $100 \mathrm{~g}$ of body wt). Inset in upper left corner represents corticosterone (B) levels measured before experiment. Values are means $\pm \mathrm{SE}$. $n$ values were as follows: sham, $10 ;$ ADRX $+0 \%$, $8 ; \quad \operatorname{ADRX}+20 \%, \quad 7 ;$ ADRX $+40 \%, 7$; ADRX $+80,8$.

TABLE 1. Effects of adrenalectomy and corticosterone replacement

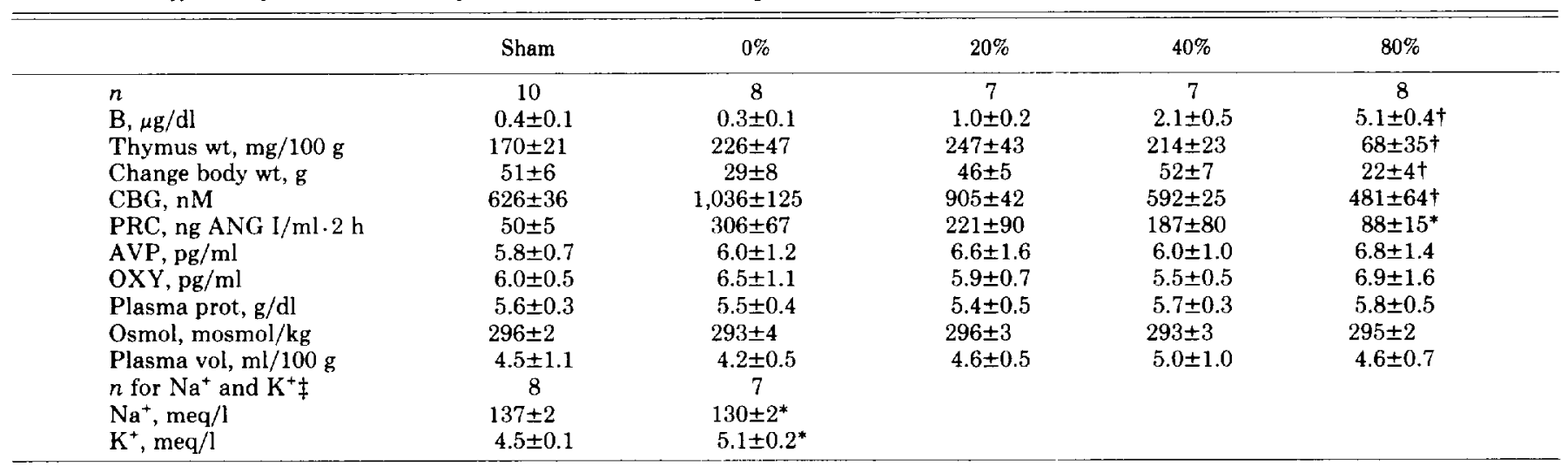

Values are means \pm SE. Plasma corticosterone (B) samples were taken in the morning, before experiment. All other plasma samples were taken the day before. Change in body wt was calculated for 7 days after adrenalectomy. $n$, no. of rats in each group. Thymus wt in mg/ $100 \mathrm{~g}$ of body wt. CBG, corticosteroid-binding globulin; PRC, plasma renin concentration; AVP, vasopressin; OXY, oxytocin; plasma prot, plasma protein; Osmol, plasma osmolality; Plasma vol, plasma volume. ${ }^{*} P<0.05$ by analysis of variance (ANOVA) except $\mathrm{Na}^{+}$and $\mathrm{K}^{+}$by $t$ test. $\dagger P<0.01$ by ANOVA. $\ddagger$ Data from animals in Fig. 1.

ered an index of baroreflex sensitivity. Table 3 demonstrates that the slopes calculated from the nitroglycerininduced decreases in MABP in the ADRX+0\%, 20\%, and $40 \%$ groups were significantly lower than the slope in the sham group. However, the slope calculated from the ADRX $+80 \%$ group was not different from the sham group. This suggests that the sensitivity of the baroreflex is lower in ADRX rats and that the $80 \%$ pellet provides adequate corticosterone replacement for this part of the plot. There were no significant differences between the slopes of any of the groups for the part of the plot generated by the phenylephrine-induced increases in MABP. There was also no difference between the $y$ intercepts of any of the groups for the part of the plot generated by phenylephrine-induced increases or nitro- glycerine-induced decreases in MABP. All regressions were shown to be highly significant by $F$ test.

MABP, heart rate, and fluid intake were measured daily, in the morning, throughout the experiment (Fig. 4). After adrenalectomy (day 3), resting MABP decreased in the ADRX+0 group. However, this decrease was not significant by ANOVA. Resting MABP values in the 20 and $40 \%$ groups were also not different from sham. However, resting MABP was significantly higher than sham in the ADRX $+80 \%$ group, suggesting an overreplacement of corticosterone. Resting heart rate was significantly elevated in the ADRX $+0 \%$ compared with all other groups. Resting heart rate in the ADRX $+20,40$, and $80 \%$ were not different from sham. Daily fluid intake did not change after adrenalectomy in the ADRX $+0 \%$ 

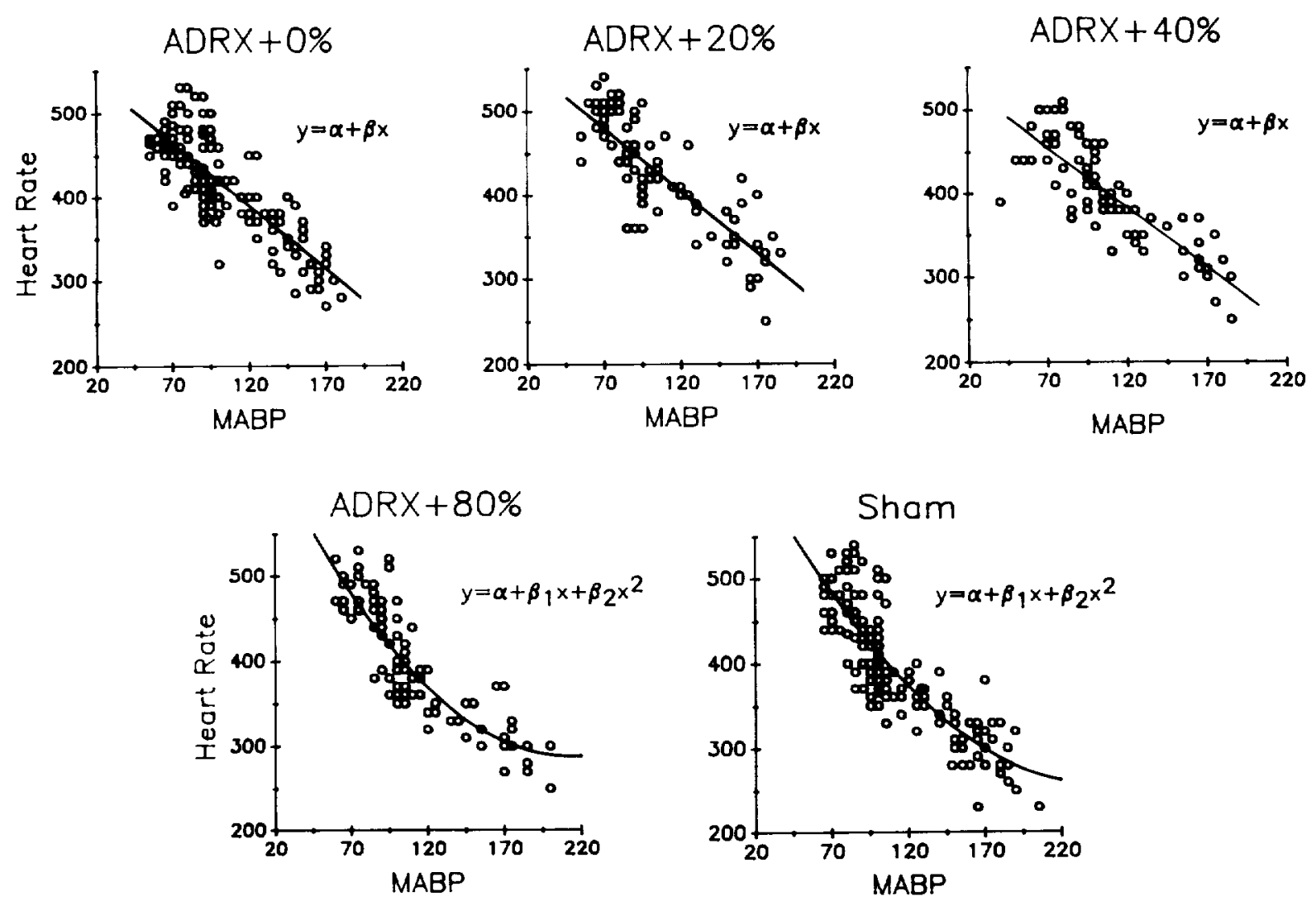

FIG. 3. Baroreflex curves generated by recording heart rate (beats $/ \mathrm{min}$ ) at varying mean arterial blood pressure (MABP, mmHg) with phenylephrine and nitroglycerin for each group. Regressions are all highly significant. ADRX, adrenalectomized.

TABLE 2. Regression coefficients for baroreflex $\left(y=\alpha+\beta_{1} x+\beta_{2} x^{2}\right)$

\begin{tabular}{lrrrrr} 
& $N$ & $\alpha$ & $\beta_{1}$ & $\beta_{2}$ & $n$ \\
\hline ADRX+0\% & 15 & $568 \pm 9.5$ & $-1.51 \pm 0.09$ & 146 \\
ADRX+20\% & 8 & $583 \pm 12.3$ & $-1.50 \pm 0.11$ & 97 \\
ADRX+40\% & 7 & $563 \pm 12.3$ & $-1.49 \pm 0.11$ & & 83 \\
ADRX $+80 \%$ & 7 & $715 \pm 39.5$ & $-4.02 \pm 0.67$ & $9.44 \times 10^{-3} \pm 2.65 \times 10^{-3}$ & 90 \\
Sham & 17 & $699 \pm 39.5$ & $-3.58 \pm 0.67$ & $7.25 \times 10^{-3} \pm 2.64 \times 10^{-3}$ & 166 \\
\hline
\end{tabular}

Values are means $\pm \mathrm{SE}$ of the estimate; $n$, no. of points in the graph; $N$, no. of rats per group. Regression coefficients for Fig. 3. ADRX, adrenalectomized.

TABLE 3. Slopes from linear regression analysis

\begin{tabular}{lll} 
& Nitroglycerin & Phenylephrine \\
\hline Sham & $-2.22 \pm 0.17$ & $-1.22 \pm 0.07$ \\
ADRX $+0 \%$ & $-1.27 \pm 0.11^{*}$ & $-1.10 \pm 0.19$ \\
ADRX+20\% & $-1.62 \pm 0.22^{*}$ & $-1.41 \pm 0.07$ \\
ADRX $+40 \%$ & $-1.27 \pm 0.20^{*}$ & $-1.12 \pm 0.07$ \\
ADRX+80\% & $-2.00 \pm 0.14$ & $-1.24 \pm 0.06$ \\
\hline
\end{tabular}

Slope calculated from nitroglycerin-induced and phenylephrine-induced changes in mean arterial blood pressure vs. changes in heart rate. ADRX, adrenalectomized. ${ }^{*} P<0.05$ compared with sham group by $t$ test.

group; however, the sham and ADRX+20, 40, and $80 \%$ groups increased their fluid intakes and were different from the ADRX+0 group.

Adrenalectomy leads to a decreased rate of body weight gain and decreased plasma $\mathrm{Na}^{+}$and an increase in thymus weight, plasma $\mathrm{CBG}, \mathrm{PRC}$, and $\mathrm{K}^{+}$(Table 1). There were corticosterone dose-dependent differences among the five treatment groups for body weight gain, thymus weight, plasma $\mathrm{CBG}, \mathrm{PRC}, \mathrm{Na}^{+}$, and $\mathrm{K}^{+}$. By NewmanKeuls analysis following ANOVA, it was found that the ADRX $+40 \%$ replacement was not different from sham treatment for body weight gain, thymus weight, and plasma CBG, suggesting that the $40 \%$ corticosterone pellet represents the best static levels of replacement. PRC was not returned to normal (sham), even with the $80 \%$ pellet. Adrenalectomy and corticosterone replacement had no effect, compared with sham, on plasma vasopressin, oxytocin, osmolality, protein, and volume (Table 1).

\section{DISCUSSION}

These data demonstrate that adrenalectomy results in a very mild impairment of vascular responsiveness to vasoconstrictor and vasodilators agents and the baroreflex in the conscious, unstressed rat and that replacement 

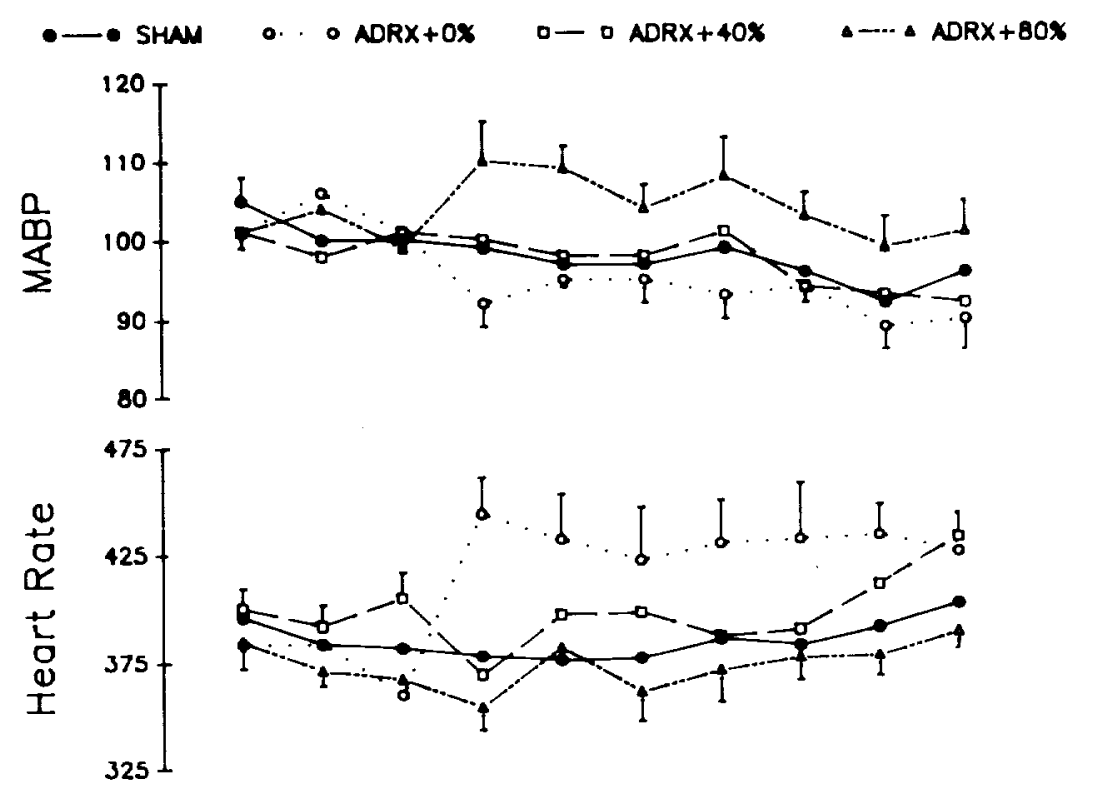

FIG. 4. Resting levels of mean arterial blood pressure (MABP, $\mathrm{mmHg}$ ), heart rate (beats $/ \mathrm{min}$ ), and fluid intake (in $\mathrm{ml} / 100 \mathrm{~g}$ body $\mathrm{wt}$ ) measured daily in the morning. Rats were given tap water ad libitum before and $0.5 \%$ saline after adrenal surgery. Values are means $\pm \mathrm{SE}$. ADRX, adrenalectomized.

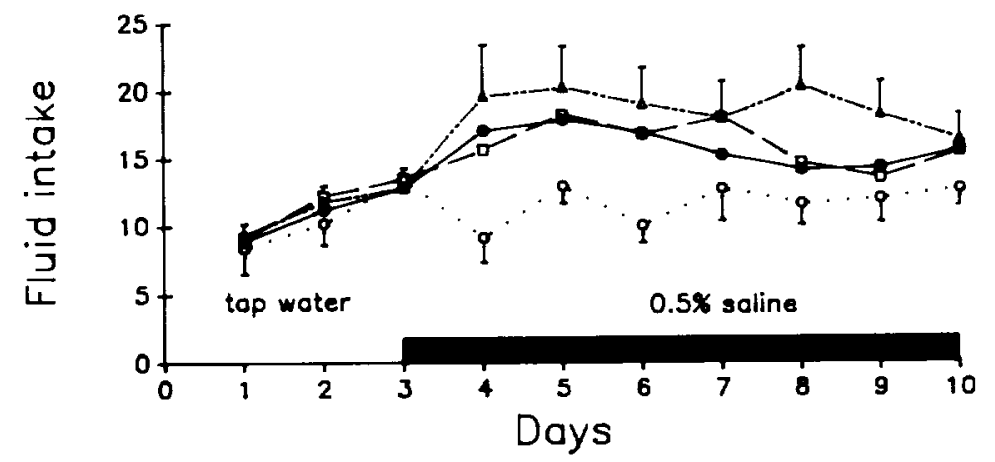

with corticosterone in the low physiological range corrects these deficits.

The replacement dose that best corrected the changes in function after adrenalectomy depended on the variable considered. Lower doses of corticosterone appeared to correct the slight decrease in basal MABP in ADRX rats (Figs. 2 and 4 ), whereas the $80 \%$ dose caused an elevation in basal MABP (Fig. 4). The replacement that best corrected the impairment in the sensitivity of the baroreflex was the $80 \%$ corticosterone pellet (Fig. 3 and Table 3 ). This was higher than the $40 \%$ pellet needed to correct the changes seen after adrenalectomy in the MABP response to phenylephrine, body weight gain, thymus weight, and CBG. The optimal replacement for vascular responsiveness varied with increasing doses of phenylephrine and nitroglycerin (Fig. 2). At low doses of phenylephrine and nitroglycerin, the curves generated in the ADRX $+20,40$, and $80 \%$ groups were not different from sham. However, at higher doses of phenylephrine, the curves generated in the ADRX $+20 \%$ and ADRX $+80 \%$ groups were lower and higher, respectively, than the sham group. Also, at higher doses of nitroglycerin, the curves generated in the ADRX+20,40, and $80 \%$ groups were lower than that in the sham group (as a function of the levels of corticosterone replaced, Fig. 2).

The results shown in Table 3 suggest that 1 ) the sensitivity of the baroreflex is depressed in the adrenal insufficiency but 2) only on one side of the baroreflex curve. The sensitivity of the reflex was significantly less in the ADRX $+0 \%$ group than in the sham group when the reflex was examined by decreasing arterial pressure. However, there was no difference in the sensitivity of the baroreflex between sham and ADRX groups when the reflex was examined by increasing arterial pressure. This strongly suggests that a factor or factors from the adrenal gland help maintain the sensitivity of the baroreflex on one side of the arterial pressure curve.

It is of interest to note that the regression equations calculated from the data from sham and ADRX +0 groups intersect at $\sim 85$ and $190 \mathrm{mmHg}$ (Fig. 3). Between these values, the sham curve dips below the curve calculated for the ADRX+0 group. This result predicts that heart rate would be higher in the ADRX + 0 group than in the sham group in this range. Coupling this with the observation that resting arterial pressure in the adrenalectomized rats tends to be lower than that in sham rats (Figs. 1 and 4), Fig. 3 predicts that resting heart rate would be higher in the ADRX +0 group than in the sham group. Examination of Fig. 4 confirms this.

In this study, adrenalectomy led to changes in body weight gain, thymus weight, and plasma CBG that were corrected by replacement with the $40 \%$ pellet, confirming the earlier finding that these variables are regulated within a narrow range of corticosterone concentrations 
$(1,5,16)$. PRC increased after adrenalectomy; the corticosterone replacement levels used in this study did not completely return renin concentration to normal, despite the fact that the $80 \%$ pellet clearly was excessive for most other variables. A recent report (30), as well as unpublished data from our laboratory suggest that physiological levels of the mineralcorticoid aldosterone return PRC to values not different from sham. Vasopressin levels in this study were slightly elevated compared with levels reported elsewhere (15). However, vasopressin concentrations may have been elevated to maintain plasma osmolality in the face of an increased $\mathrm{NaCl}$ intake from the drinking fluid $(0.5 \%$ saline). Plasma volume tended to be lower in the ADRX +0 group, which is in agreement with others (17); however, the difference from sham was not significant.

Glucocorticoid replacement is crucial in the treatment of patients with adrenal crisis. In humans, the clinical signs of adrenal insufficiency (Addison's disease) are weight loss, hypotension, and a weak and feeble heart ( 3 , 11). Abnormally high levels of glucocorticoids (Cushing's syndrome) are equally debilitating (13) and may contribute to or accelerate certain aspects of aging and increase the sensitivity of the organism to neurological insults (22). This report and others $(1,5,16)$ have shown in the rat that too much or too little corticosterone will overor undercorrect glucocorticoid-regulated variables (basal MABP, vascular responsiveness, baroreflex, body weight, thymus weight, and $C B G$ ) and suggest that very accurate replacement of corticosterone is necessary to restore these variables in adrenalectomized rats to normal. The importance of the glucocorticoids is further emphasized by a recent study showing that replacement with physiological levels of aldosterone, alone, does not correct the change in body weight, basal MABP, or vascular responsiveness to norepinephrine or angiotensin after adrenalectomy. However, replacement with the synthetic glucocorticoid dexamethasone does (30).

The loss of vascular responsiveness to vasoactive agents after removal of adrenal steroids and the subsequent recovery after glucocorticoid treatment has been explained by a combination of mechanisms. Glucocorticoids have been reported to inhibit the action of catechol $O$-methyltransferase and monoamine oxidase in effector tissues and they also decrease the ability of the neuronal and extraneuronal uptake systems to function properly $(4,9)$. This would allow accumulation of catecholamines in synaptic junctions and could possibly explain the hyporesponsive vasculature in ADRX rats and the hyperresponsive vasculature in ADRX rats with overreplacement of corticosterone (Fig. 2). There are also reports that adrenalectomy and glucocorticoid replacement change the density of adrenergic receptors in various tissues. However, it is difficult to draw general conclusions about these data, because the effects change from tissue to tissue (for review see Ref. 9). Another mechanism by which glucocorticoids may affect vascular responsiveness is through central modulation of the baroreflex. Recently, glucocorticoid receptors were found in cells located in medullary structures known to mediate baroreceptor signals and regulate sympathetic output
(11). It is to be noted, however, that the degree of impairment in vascular responsiveness to pressor and depressor agents found in adrenalectomized rats in this study was very slight. We ascribe this result to the fact that the rats were studied in the conscious state, at least 5 days after any surgery or stress to the animals had been administered.

The circadian rhythm for corticosterone in rats resulted in values that are low in the morning $(0.5 \pm 0.1$ $\mu \mathrm{g} / \mathrm{dl})$ and high in the evening $(9.0 \pm 2.3 \mu \mathrm{g} / \mathrm{dl})$. Because the restoration of vascular responsiveness and the baroreflex required concentrations that are higher than those measured in the morning (40 and $80 \%$ pellet), it is possible that the normal diurnal elevation of corticosterone in the evening is necessary to maintain vascular responsiveness and normal baroreflexes in sham rats. We have recently reported that stress-induced adrenocorticotropic hormone secretion is normalized in adrenalectomized rats by phasic but not constant corticosterone replacement (12) and have speculated that transient elevations in corticosterone are required for adequate daily occupancy of glucocorticoid receptors in brain. Because glucocorticoid receptors have been reported to be localized in medullary regions that control the cardiovascular system (11), it is logical to propose that transient daily occupancy of these receptors could also normalize responses in the cardiovascular system of ADRX rats.

We thank Caren Cascio and Dr. Robert W. Kuhn for performing the corticosterone and corticosteroid-binding globulin assays. Our special thanks to Dr. Vojtech Licko, Cardiovascular Research Institute, Univ. of California, San Francisco, for his expertise in regression analysis and his interest in the problem.

This work was supported by National Institutes of Health Grants KD-28172, HL-29714, and NCC2-350. K. Kaship was supported by The American Heart Association, California Affiliate, student research program.

Address for reprint requests: D. N. Darlington, Dept. of Physiology, Box 0444, Univ. of California, San Francisco, San Francisco, CA 94143.

Received 7 July 1988; accepted in final form 21 December 1988.

\section{REFERENCES}

1. Akana, S. F., C. S. Cascio, J. Shinsako, and M. F. Dallman. Corticosterone: narrow range required for normal body and thymus weight and ACTH. Am. J. Physiol. 249 (Regulatory Integrative Comp. Physiol. 18): R527-R532, 1985.

2. Cleghorn, R. A. Cardiovascular failure in experimental adrenal insufficiency: a historical revival. Perspect. Biol. Med. 27: 135-155, 1983.

3. Cullen, D. R., J. P. D. Reckless, and E. H. Mclaren. Clinical disorders involving adrenocortical insufficiency and overreactivity. In: General Comparative and Clinical Endocrinology of the Adrenal Cortex, edited by I. C. Jones and I. W. Henderson. New York: Academic, 1980 , vol. 3 , p. 57-77.

4. Dailely, J. W., and T. C. Westrall. Effects of adrenalectomy and adrenal steroids on norepinephrine synthesis and monoamine oxidase activity. Eur. J. Pharmacol. 48: 383-391, 1978.

5. Dallman, M. F., S. F. Akana, C. S. Cascio, D. N. Darlington, L. JACOBSON, AND N. LEVIN. Regulation of ACTH secretion: variations on a theme of B. Recent Prog. Horm. Res. 43: 113-173, 1987.

6. Darlington, D. N., J. Shinsako, and M. F. Dallman. Paraventricular lesions: hormonal and cardiovascular responses to hemorrhage. Brain Res. 439: 289-301, 1988.

7. Fowler, J. L. A., AND R. A. ClEGHORN. The response of splanchnic blood vessels and of the small intestine to vasoconstrictor 
influences in adrenal insufficiency in the cat. Am. J. Physiol. 137: $371-379,1942$.

8. FRITZ, I., AND R. LEvine. Action of adrenal cortical steroids and norepinephrine on vascular responses of stress in adrenalectomized rats. Am. J. Physiol. 165: 456-465, 1951.

9. Gibson, A. The influence of endocrine hormones on the autonomic nervous system. J. Auton. Pharmacol. 1: 331-358, 1981.

10. Harfstrand, A., K. Fuxe, A. Cintra, L. F. Agnati, I. Zini, A. Wikstrom, S. OKRET, Z. YU, M. Goldstein, H. STEINBUSCh, A VERHOFSTAD, AND J. GuSTAFsSON. Glucocorticoid receptor immunoreactivity in monoaminergic neurons of rat brain. Proc. Natl. Acad. Sci. USA 83: 9779-9783, 1986.

11. Irvine, W. J., A. D. Toft, AND C. M. FeEk. Addison's disease. In: The Adrenal Gland, edited by V. H. T. James. New York: Raven, 1979, p. 131-164.

12. Jacobson, L., S. F. Akana, C. S. Cascio, J. Shinsako, and M. F. DALlmaN. Circadian variations in plasma corticosterone permit normal termination of ACTH responses to stress. Endocrinology 122: $1343-1348,1988$.

13. JEFFCOATE, W. J., AND C. R. W. EDWaRDs. Cushing's syndrome: pathogenesis, diagnosis, and treatment. In: The Adrenal Gland edited by V. H. T. James. New York: Raven, 1979, p. 165-195.

14. Kalsner, S. Steroid potentiation of responses to sympathomimetic amines in aortic strips. Br. J. Pharmacol. 36: 582-593, 1969.

15. Keil, L. C., L. M. Rosella-Dampman, S. Emmert, O. Chee, AND J. Y. SUMMY-LONG. Enkephalin inhibition of angiotensinstimulated release of oxytocin and vasopressin. Brain Res. 297: $329-336,1984$

16. Levin, N., S. F. Akana, L. Jacobson, R. W. Kuhn, P. K. Sitteri, AND M. F. Dallman. Plasma adrenocorticotropin is more sensitive than transcortin production or thymus weight to inhibition by corticosterone in rats. Endocrinology 121: 1104-1110, 1987.

17. Moses, A. M. Influence of adrenal cortex on body water distribution in rats. Am. J. Physiol. 208: 662-665, 1965.

18. Ramey, E. R., M. S. Goldstein, and R. Levine. Action of norepinephrine and adrenal cortical steroids on blood pressure and work performance of adrenalectomized dogs. Am. J. Physiol. 105: $450-455,1951$.
19. RAYmoure, W. J., AND R. K. KUHN. A homologous radioimmunoassay for rat corticosteroid binding globulin. Endocrinology 112: $1091-1097,1983$

20. Reidenberg, M. M., E. A. Ohler, R. W. Seyy, and C. Harakal. Hemodynamic changes in adrenalectomized dogs. Endocrinology 72: 918-923, 1963.

21. Ross, E. J. Functional relationship between adrenal medullary and cortical hormones in man. Q. J. Med. 30: 285-296, 1961

22. Sapolsky, R. M., L. C. KREy, and B. S. McEwen. The neuroen docrinology of stress and aging: The glucocorticoid cascade hy potheses. Endocr. Rev. 7: 284-301, 1986.

23. Schomig, A., B. Luth, R. Dietz, and F. Gross. Changes in vascular smooth muscle sensitivity to vasoconstrictor agents induced by corticosteroids, adrenalectomy and differing salt intake in rats. Clin. Sci. Mol. Med. 51, Suppl. 3: 61s-63s, 1976.

24. Small, H. S., S. W. Weitzmer, and G. G. Nahas. Cardiovascular effects of levarterenol, hydrocortisone and hemisuccinate, and al dosterone in the dog. Am. J. Physiol. 165: 1025-1028, 1959.

25. Stockigt, J. R., R. D. Collins, and E. G. Biglieri. Determination of plasma renin concentration by angiotensin I immunoassay. Diagnostic import of precise measurement of subnormal renin in hyperaldosteronism. Circ. Res. 28/29, Suppl. II: 175-187, 1971.

26. TANZ, R. D. Studies on the action of cortisone acetate on isolated cardiac tissue. J. Pharmacol. Exp. Ther. 128: 168-174, 1960.

27. Wade, C. E., P. Bie, L. C. KeIL, and D. J. Ramsay. Osmotic control of plasma vasopressin in the dog. Am. J. Physiol. 243 (Endocrinol. Metab. 6): E287-E292, 1982.

28. Webb, W. R., I. U. Degerli, J. D. Hardy, and M. Unal. Cardiovascular responses in adrenal insufficiency. Surgery 58: 273-282, 1965.

29. Wilkinson, C., J. Shinsako, and M. F. Dallman. Return of pituitary-adrenal function after enucleation or transplantation diurnal rhythms and responses to ether. Endocrinology 109: 162 $169,1981$.

30. Yagil, Y., AND L. R. KrakofF. The differential effects of aldosterone and dexamethasone on pressor responses in adrenalectomized rats. Hypertension Dallas 11: 174-178, 1988.

31. ZAR, J. H. Polynomial regression. In: Biostatistical Analysis (2nd ed.). Englewood Cliffs, N.J: Prentice-Hall, 1984, p. 361-366. 